\title{
Using the curve moment and the PSO-SVM method to diagnose downhole conditions of a sucker rod pumping unit
}

\author{
Li Kun, Gao Xianwen*, Tian Zhongda and Qiu Zhixue \\ College of Information Science and Engineering, Northeastern University, Shenyang, Liaoning 110819, China \\ (C) China University of Petroleum (Beijing) and Springer-Verlag Berlin Heidelberg 2013
}

\begin{abstract}
Downhole working conditions of sucker rod pumping wells are automatically identified on a computer from the analysis of dynamometer cards. In this process, extraction of feature parameters and pattern classification are two key steps. The dynamometer card is firstly divided into four parts which include different production information according to the "four point method" used in actual oilfield production, and then the moment invariants for pattern recognition are extracted. An improved support vector machine (SVM) method is used for pattern classification whose error penalty parameter $C$ and kernel function parameter $g$ are optimally chosen by the particle swarm optimization (PSO) algorithm. The simulation results show the method proposed in this paper has good classification results.
\end{abstract}

Key words: Sucker rod pumping unit, diagnosis of downhole conditions, dynamometer card, curve moment, support vector machine, particle swarm optimization

\section{Introduction}

In actual oilfield production, it is difficult to know the real downhole working conditions of oil rod pumping systems working thousands of meters underground. The conventional methods rely on engineers to analyze the cards collected from the dynamometer measuring the force on the sucker rod pump. Traditional manual interpretation of the card shapes can encounter some problems, such as influence by subjective factors, no real-time analysis, and high cost. With the development of automation technology in petroleum production, many jobs relying on people have not yet met the actual production requirements. So, it is important to use the machine learning method to replace the manual task so as to increase the working efficiency. Many advanced analytical methods have been used in diagnosis of downhole conditions of sucker rod pumping units, such as expert systems (Derek et al, 1988; Martinez et al, 1993), artificial neural networks (Rogers et al, 1990; Xu et al, 2007; de Souza et al, 2009; Wu et al, 2011), rough set theory (Wang and Bao, 2008), filter techniques (Li et al, 2010) and frequency spectrum analysis (He et al, 2008). However, the expert system and the rough set have a single way of knowledge expression and reasoning strategy, the artificial neural network needs a large number of training samples and application of the filter technique and frequency spectrum analysis have some limitations. The support vector machine

*Corresponding author. email: gaoxianwen@ise.neu.edu.cn Received March 7, 2012
(SVM) technique is a good pattern recognition method as it only needs a small number of training samples and has good generalization ability, which is widely used in many research fields (Ganapathiraju et al, 2004; Campbell et al, 2006; Nath and Shevade, 2006; Widodo and Yang, 2007; Chowdhury et al, 2011; Mountrakis et al, 2011; Wang et al, 2011; Li et al, 2012). It has been used to solve diagnosis faults in sucker rod pumping units in many instances (Shi et al, 2004; Li et al, 2006; Tian et al, 2007a; 2007b). However, more in-depth work should be undertaken in selection of feature parameters and classification performance. So, this study firstly uses the moment curve method to extract the features of typical dynamometer cards, and then uses the improved SVM method for pattern classification which is combined with particle swarm optimization (PSO) algorithm to choose the best error penalty factor $C$ and kernel function parameter $g$ so as to improve classification efficiency. The final examples show that the proposed method has good classification results for fault diagnosis of sucker rod pumping units.

\section{Downhole dynamometer card in sucker rod pumping wells}

A one-dimensional wave equation with viscous damping which describes dynamic changes of a sucker rod string is used to calculate the downhole dynamometer card in a sucker rod pumping unit, which can truly reflect working conditions of the subsurface pump as it may eliminate effects of the deformation, viscous resistance, vibration and inertia of the sucker rod string (Gibbs and Neely, 1966). Our study uses 
the Fourier coefficient method to solve the one-dimensional wave equation proposed by Gibbs. Because the dynamic load function and the displacement function of the polished rod are given in numerical forms, the Fourier coefficients may be determined by approximate numerical integration (Chen, 1988). The calculated result of the pump dynamometer card is shown in Fig. 1.

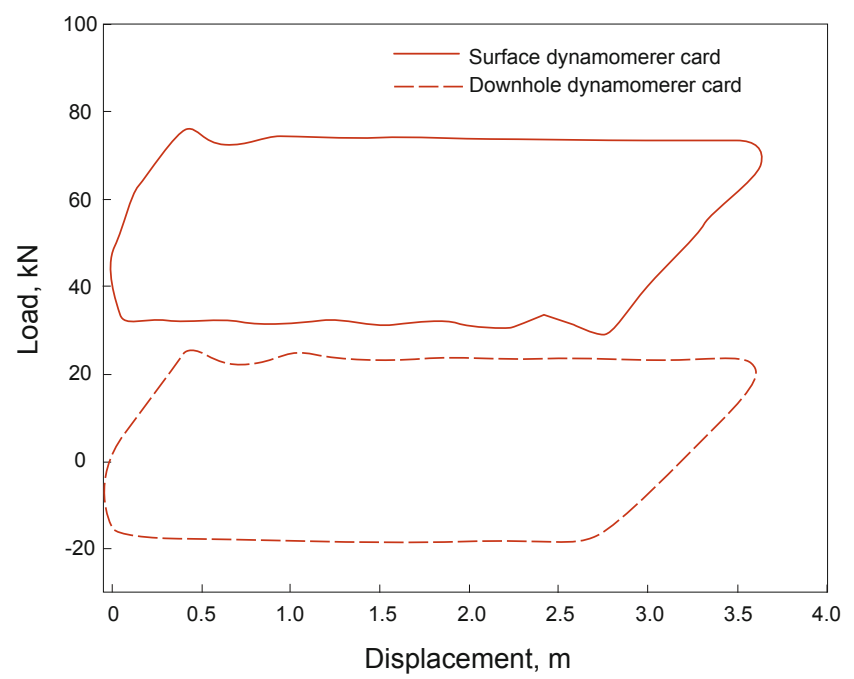

Fig. 1 Calculated downhole dynamometer card from a measured surface dynamometer card

\section{Feature extraction of the downhole dynamometer card based on the curve moment}

The data need to be normalized before feature extraction as the data precision and dimension of the dynamometer cards in different sucker rod pumping wells may not be the same, which is transformed into $[0,1]$ interval. The normalization formula is as follows:

$$
\begin{aligned}
& \bar{x}=\frac{x_{i}-x_{\min }}{x_{\max }-x_{\min }} \\
& \bar{y}=\frac{y_{i}-y_{\min }}{y_{\max }-y_{\min }}
\end{aligned}
$$

where $x_{i}$ and $y_{i}$ represent the displacement and load data; $\bar{x}$ and $\bar{y}$ represent the normalized data.

Known from the principle of the dynamometer card, the geometric features of all types of dynamometer cards are characterized by changes of the valve open position, that is, the four corners of the parallelogram. So, it is particularly important to determine the fully open and closed positions of the valve, which is the four-point method commonly used in pump fault diagnosis (Liang and Li, 2011). Taking the theoretical dynamometer card for example, its partition is shown in Fig. 2.

Seen from the diagram, the dynamometer card is divided into four parts, lower left, upper left, upper right and lower right, respectively, which contains $A, B, C$ and $D$ points

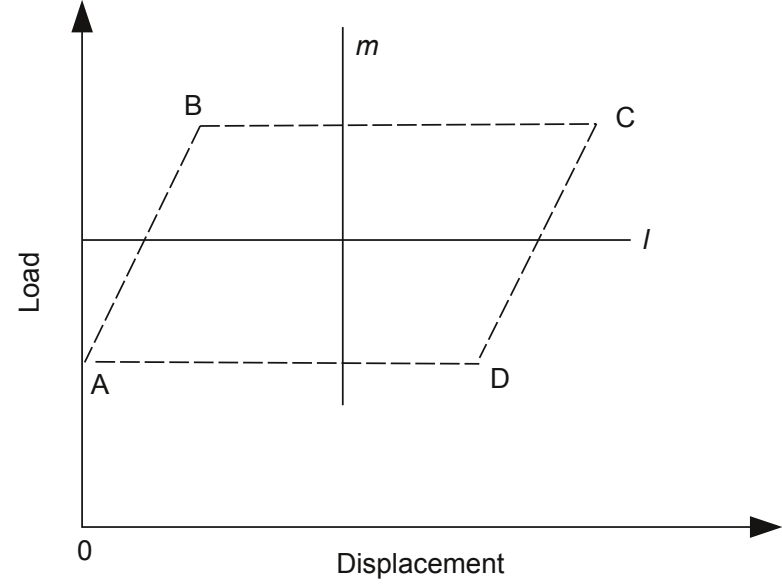

Fig. 2 Partition of the theoretical dynamometer card

reflecting the working states of the pumping unit. The equations of straight lines $l$ and $m$ are:

$$
\begin{aligned}
& l=\frac{1}{2}\left(L_{\max }+L_{\text {min }}\right) \\
& m=\frac{1}{2} S
\end{aligned}
$$

where $L_{\max }$ and $L_{\min }$ represent the maximum load and the minimum load respectively; $S$ represents the stroke.

The partition results of one dynamometer card are shown in Fig. 3.

The curve moment theory (Chen, 1993) is introduced to extract the moment features of the dynamometer card in this paper.

Geometric moment and geometric central moment of the curve moment are defined as follows:

$$
\begin{aligned}
& m_{p q}=\sum_{i=1}^{M} \int_{l_{i(s)}} x^{p} y^{q} \mathrm{~d} s \\
& \mu_{p q}=\sum_{i=1}^{M} \int_{l_{i(s)}}(x-\bar{x})^{p}(y-\bar{y})^{q} \mathrm{~d} s
\end{aligned}
$$

where $M$ is the number of the curve segments; $l_{i(s)}$ is the $i$ st curve segment; $\bar{x}=m_{10} / m_{00} ; \bar{y}=m_{01} / m_{00}$; $p, q=0,1,2, \cdots$.

Research by Wolfson in 1995 proves that the central moment $\mu_{p q}$ of the contour moment is not affected by movement, rotation, zoom (magnification and minimization) and other transformations. As the curve moment is an improved form of the contour moment, so the central moment is also not affected by movement, rotation, zoom and other transformations.

The feature extraction of the downhole dynamometer card is conducted as follows.

Each part after partition is a curve composed of discrete points $\left(x_{i}, y_{i}\right), i=1,2, \cdots, N$. Its $p+q$ rank curve moment $m_{p q}$ is defined as: 

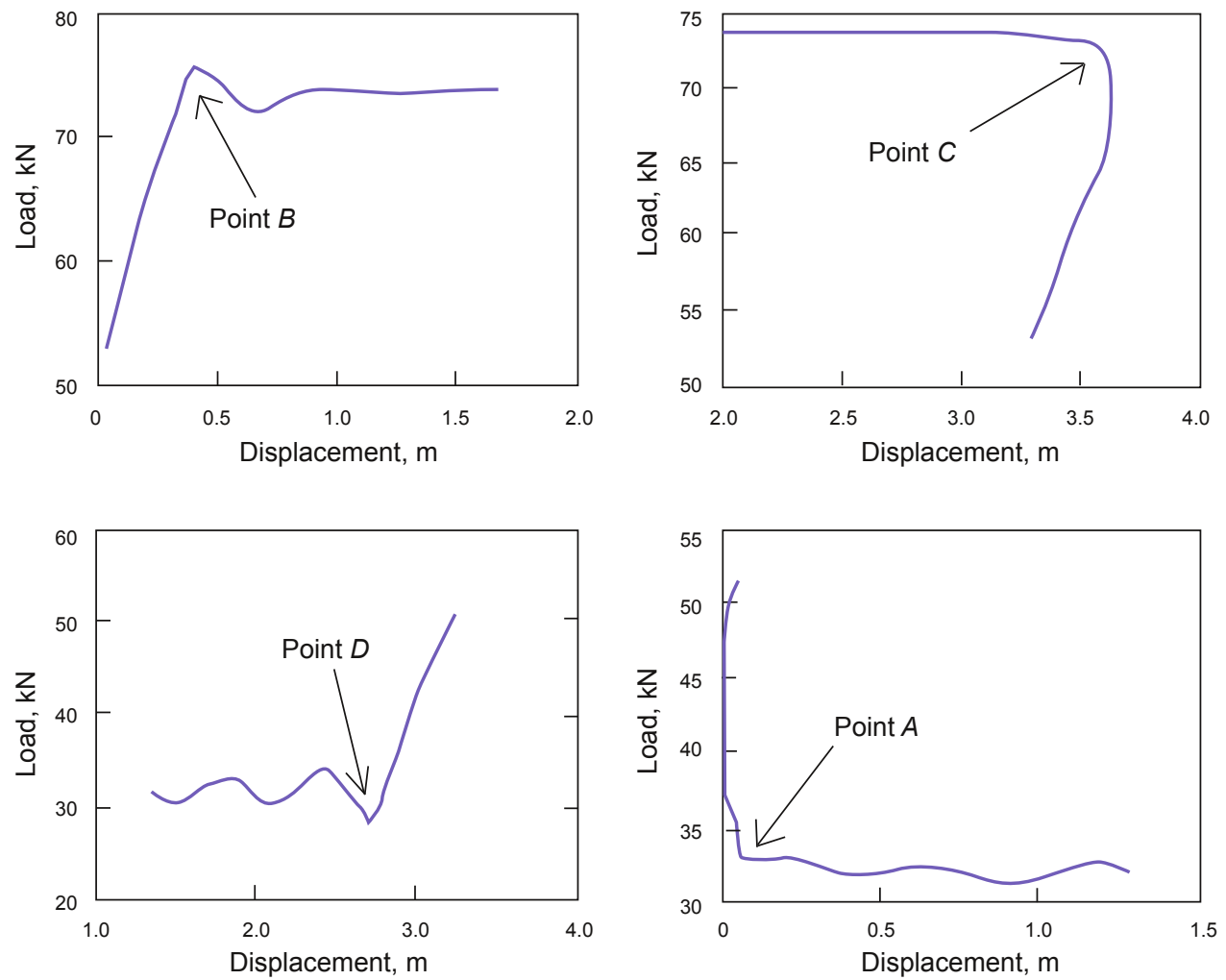

Fig. 3 Four parts of the dynamometer card

$$
m_{p q}=\sum_{i=1}^{N} x_{i}^{p} y_{i}^{q} \Delta l_{i}
$$

where $x_{i}$ is the horizontal coordinate of the discrete point; $y_{i}$ is the vertical coordinate of the discrete point; $i$ is the $i$ st discrete point in the curve edge; $N$ is the total number of the discrete points, $\Delta l_{i}$ is the straight-line distance between two adjacent discrete points, $\Delta l_{i}=\sqrt{\left(x_{i}-x_{i-1}\right)^{2}+\left(y_{i}-y_{i-1}\right)^{2}}$, $p, q=0,1,2, \cdots$.

The corresponding $p+q$ rank central moment is defined as:

$$
\mu_{p q}=\sum_{i=1}^{N}\left(x_{i}-\bar{x}\right)^{p}\left(y_{i}-\bar{y}\right)^{q} \Delta l_{i}
$$

where point $(\bar{x}, \bar{y})$ is the barycentric coordinates of the curve.

Calculating the central moment of each rank:

$$
\begin{aligned}
& \mu_{00}=m_{00} \\
& \mu_{10}=0 \\
& \mu_{01}=0 \\
& \mu_{11}=m_{11}-\bar{y} m_{10} \\
& \mu_{20}=m_{20}-\bar{x} m_{10} \\
& \mu_{02}=m_{02}-\bar{x} m_{01}
\end{aligned}
$$

$$
\begin{aligned}
& \mu_{30}=m_{30}-3 \bar{x} m_{20}+2 m_{10} \bar{x}^{2} \\
& \mu_{03}=m_{03}-3 \bar{y} m_{02}+2 m_{01} \bar{y}^{2} \\
& \mu_{12}=m_{12}-2 \bar{y} m_{11}-\bar{x} m_{02}+2 \bar{y}^{2} m_{10} \\
& \mu_{21}=m_{21}-2 \bar{x} m_{11}-\bar{y} m_{02}+2 \bar{x}^{2} m_{01}
\end{aligned}
$$

It needs to be explained that each rank moment of the curve has its physical significance. According to the definition of the moment, $m_{00}=\mu_{00}=\Delta l_{i}$, the zero rank moment and the zero rank central moment of the curve denote its length; one rank moment $m_{10}$ and $m_{01}$ are used to determine the gray centroid of the curve; two rank central moment $\mu_{20}$, $\mu_{11}$ and $\mu_{02}$ are used to measure the size and direction of the curve, which are also called moment of inertia; three rank central moment $\mu_{30}$ and $\mu_{03}$ describe the graphic asymmetry. Asymmetry is a classical statistic used to measure the degree of deviation of the mean distribution. $\mu_{03}$ represents the asymmetry measurement of the curve about the vertical axis; $\mu_{30}$ represents the asymmetry measurement of the curve about the horizontal axis. If the curve is completely symmetrical, then value of the $\mu_{30}$ and $\mu_{03}$ are zero. The central moment of each rank are used to calculate the invariant curve moment. A total of 28 vectors form one vector $\varphi=\left[\varphi_{1}, \varphi_{2}, \cdots, \varphi_{28}\right]$. This has two advantages: firstly, each feature vector is an invariant moment which is from linear or nonlinear combinations of each rank curve moment, so each feature vector has its geometric meaning; secondly, more importantly, 28 feature 
vectors by partition calculation can describe the details of the pump dynamometer card accurately.

Seven invariant moment ranges vary greatly in their calculation. So, considering the size of the invariant moments and the structural characteristic of the following pattern recognition, a total of 28 feature parameters in four parts of the downhole dynamometer card are revised to adjust its range.

The modification formula of the invariant moment is:

$$
\varphi_{i}^{\prime}=|\lg | \varphi_{i}||
$$

where $i=1,2, \cdots, 28$.

\section{Pattern classification of downhole dynamometer cards based on PSO-SVM}

SVM is a pattern classification method which only needs a small number of training samples, and has good generalization ability (Chapelle et al, 2002). We use an improved SVM method for pattern recognition of downhole dynamometer cards in oil rod pumping units.

An important step of designing SVM is to choose the kernel function and kernel parameters. Research by Chapelle et al, 2002, indicates that, when it lacks prior knowledge of the process, the results achieved by the Gauss kernel function are better than other kernel functions. Based on this, this work adopts a radial basis function as the kernel function of SVM.

For classification problems, parameters affecting classification results of SVM are mainly two: error penalty parameter $C$ and kernel function parameter $g$. The error penalty parameter $C$ adjusts the ratio of the confidence interval of the learning machine and experiences risk in the determined data subspace in order to optimize the learning machine's generalization ability, which is not the same in different data subspaces. The kernel function parameter $g$ mainly affects the complexity of sample data distribution in the high feature space. Changes of the kernel function actually change the mapping function and thereby change the complexity (number of dimensions) of sample data distribution in the data subspace. For a given kernel function (kernel function type and kernel parameters have been identified), it corresponds to a data subspace with determined dimensions, and limits the complexity of the constructed "optimal classification surface of the most complex" in this data subspace, and also determines the VC dimension (VC dimension is a measure of function class, and the higher $\mathrm{VC}$ dimension denotes the more complexity of a problem) of the optimal SVM that the subspace corresponds to.

This study applies a particle swarm optimization (PSO) algorithm to choose the best $C$ and $g$ which play important roles in the SVM classification method. A PSO algorithm is an optimization algorithm based on swarm intelligence in the computational intelligence field, which was first proposed by Kennedy, Eberhart and Shi (Eberhart and Kennedy, 1995; Kennedy and Eberhart, 1995; Shi and Eberhart, 1998). Its basic concept comes from the research into birds' predation action. That is, when birds are finding food, the most simple and effective way of each bird to find food is to search the surroundings of the bird that has the shortest distance to food currently. Its algorithm steps are as follows:

1) Initialize the positions of a set of particles. The position vector of each particle is a real number between 0 and 1,000. Determine $c_{1}, c_{2}$ and calculate $k$ and the fitness of each particle with a fitness function. The initial fitness is regarded as the particle's personal optimal solution to search for the global optimal solution.

2) Calculate the update speed and the updated position of each particle.

3) Use the evaluation function to evaluate all of the particles. When a particle's current evaluation value is better than its historical evaluation value, the current evaluation value is regarded as the historical optimal evaluation value, and the current position vector is credited as optimal position vector.

4) Search for the global optimal solution. If its value is better than the current historical optimal solution, update it; end the search if it satisfies the termination condition, otherwise go to step 2 to a new round of search.

A flow chart of the selection of SVM parameters $C$ and $g$ based on PSO algorithm is shown in Fig. 4.

\section{Simulation}

The simulation in this paper is realized by the library for support vector machines (LibSVM). Selecting 128 typical dynamometer cards, in which, fault type 1 stands for normal working of the pumping unit, 14 samples; fault type 2 stands for gas influence, 20 samples; fault type 3 stands for feed liquid failure, 24 samples; fault type 4 stands for sucker rod breakage, 10 samples; fault type 5 stands for oil of high viscosity, 10 samples; fault type 6 stands for travelling valve leakage, 10 samples; fault type 7 stands for pump bumping in bottom dead position, 10 samples; fault type 8 stands for pump bumping in top dead position, 10 samples; fault type 9 stands for standing valve leakage, 10 samples; fault type 10 stands for sand production, 10 samples.

The input of each sample is 28 invariant moments, the output is fault type, and the coding modes are respectively $1-$ fault type 1,2-fault type 2, .., 10-fault type 10 .

Firstly, the SVM with random parameters $C=2$ and $g=0.01$ is used to do fault pattern classification. In this simulation, the sample set is divided into two parts, of which 88 dynamometer cards in the training set and 40 dynamometer cards in the testing set. The results are shown in Figs. 5 and 6.

Figs. 5 and 6 indicate that the classification accuracy rate is $77 \%$ in training samples and $70 \%$ in testing samples. The accuracy rates are not satisfactory.

In order to improve the accuracy rate, the methods of cross validation $(\mathrm{CV})$ and "grid search" are used to optimally select parameters $C$ and $g$.

The specific simulation is as follows: firstly according to the $\mathrm{K}$-fold cross validation (K-CV) method the training samples are divided into four groups, then the "grid search" method is used to test different parameters $C$ and $g$ in the cross validation process in order to obtain different accuracy rates. Finally, the parameter which has the best accuracy rate is chosen as the optimal one. We use the exponential growth 

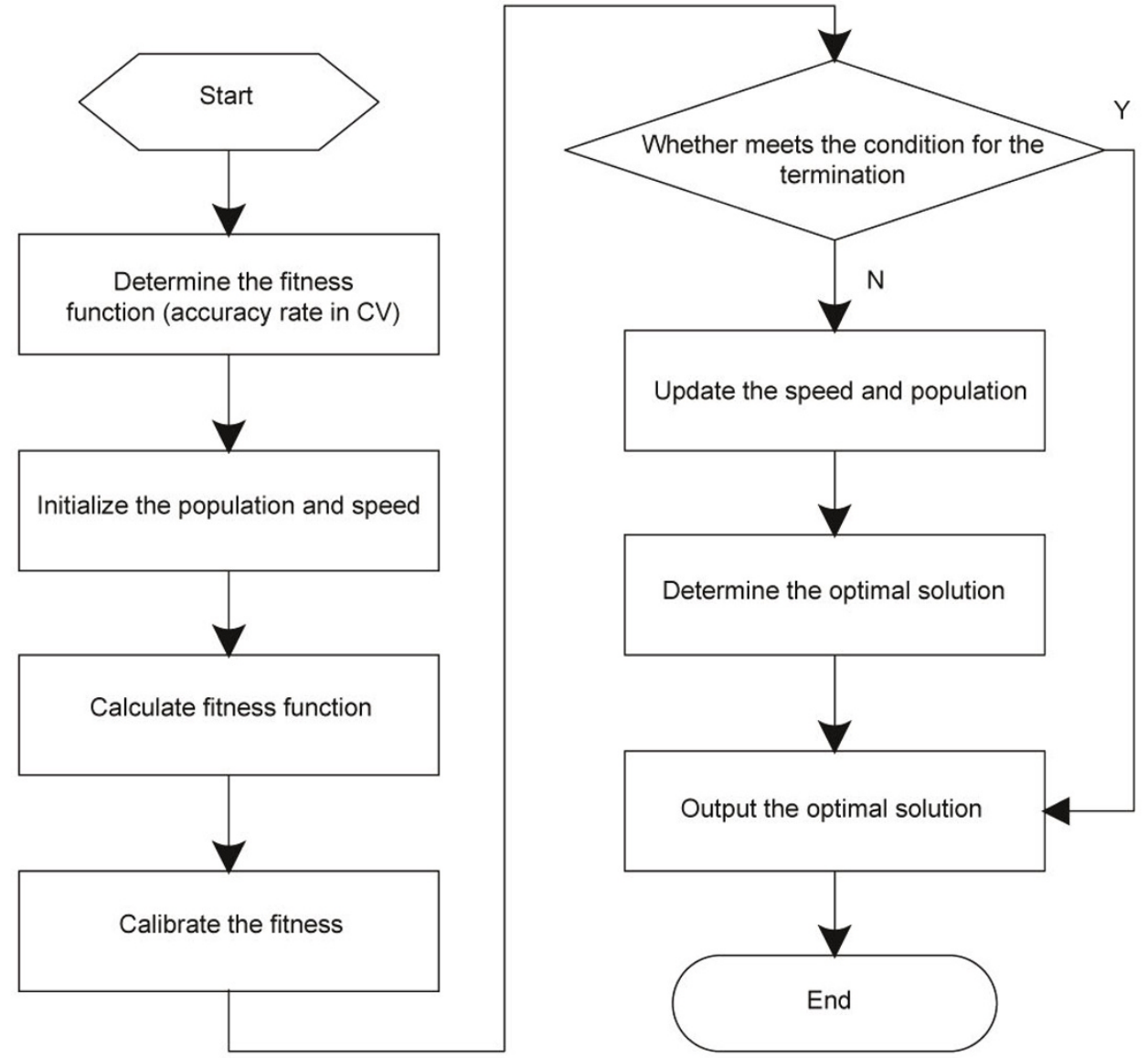

Fig. 4 Flow chart of the selection of SVM parameter $C$ and $g$ based on the PSO algorithm

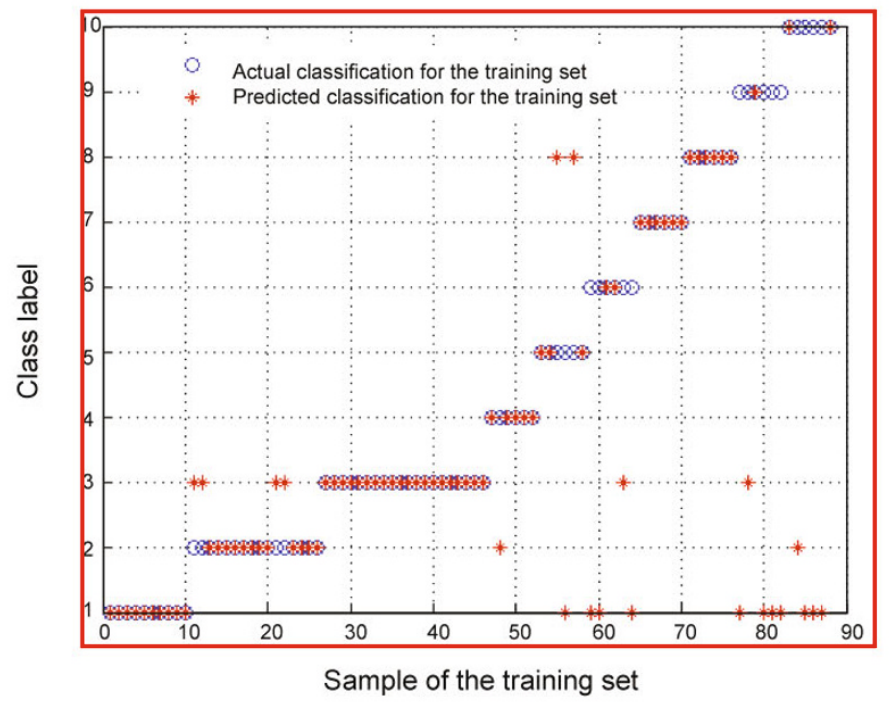

Fig. 5 Actual classification and predicted classification of training samples when $C=2, g=0.01$

method to find the best $C$ and $g$ by increasing or decreasing the values of them. In order to expand the searching scope, it uses $C=2^{-10}, 2^{-9}, 2^{-8}, \cdots, 2^{9}, 2^{10}$ and $g=2^{-10}, 2^{-9}, 2^{-8}, \cdots, 2^{9}, 2^{10}$. It can be regarded as searching some grid points in the specified range on an $x-y$ plane, and the $x$ and $y$ coordinates of each point are regarded as the values of $C$ and $g$ after conversion (such as $2^{x}$ and $2^{y}$ ). The group with the smaller $C$ is selected

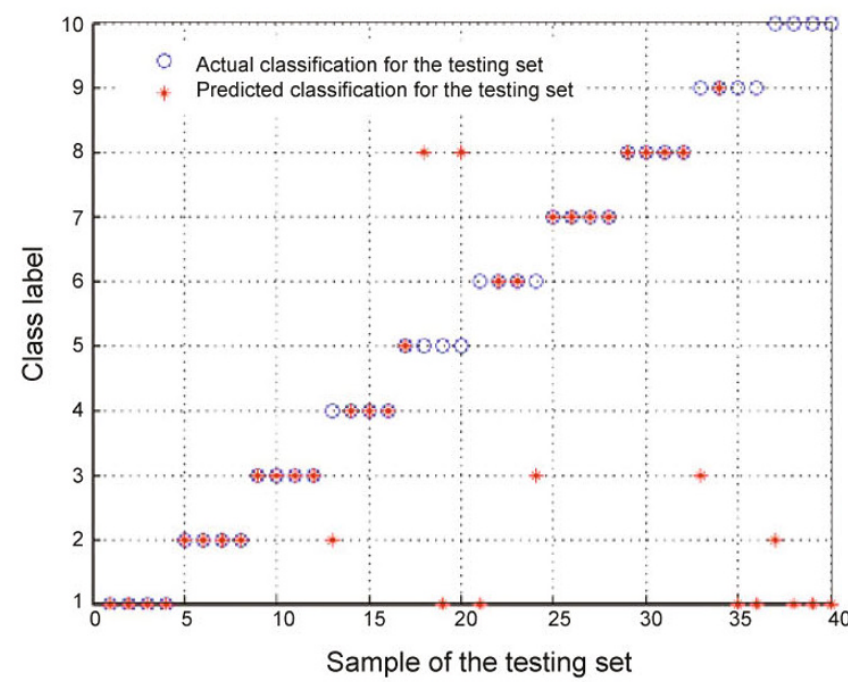

Fig. 6 Actual classification and predicted classification of testing samples when $C=2, g=0.01$

as the result if a number of groups with the same accuracy rate exist.

According to the above steps, the contour map of the parameter selection results is shown in Fig. 7.

Fig. 7 indicates that the higher classification accuracy of the training samples is in the interval $C=\left[2^{-2}, 2^{22}\right]$ and $g=\left[2^{-2}\right.$, $\left.2^{2}\right]$. In order to find the best $C$ and $g$, the ranges of $C$ and $g$ are 
re-selected as $C=2^{-2}, 2^{-15}, 2^{-1}, \cdots, 2^{1.5}, 2^{2}$ and $g=2^{-2}, 2^{-15}, 2^{-1}, \cdots$, $2^{1.5}, 2^{2}$. The results are shown in Fig. 8.

Thus, the best parameters $C=0.35355$ and $g=2$ are used to verify 40 testing samples, and the results are shown in Fig. 9.

The CV method can be used effectively to avoid occurrence of overfitting and underfitting, and obtain the optimal parameters in a certain extent. The classification accuracy rate of the testing samples reaches a relatively high level, $85 \%$.

In the parameter optimization, the target value obtained by the $\mathrm{CV}$ method is the classification accuracy rate of the training samples, but not the classification accuracy rate of the testing samples which better reflects the generalization ability of SVM. This paper applies the PSO optimization algorithm to select the parameters $C$ and $g$.

The selection of the corresponding parameters is as follows:

Iteration number $\mathrm{MaxGen}=200$; population number SizePop $=20$; parameters $c_{1}=1.5, c_{2}=1.7$; inertia factor $w=1$; the

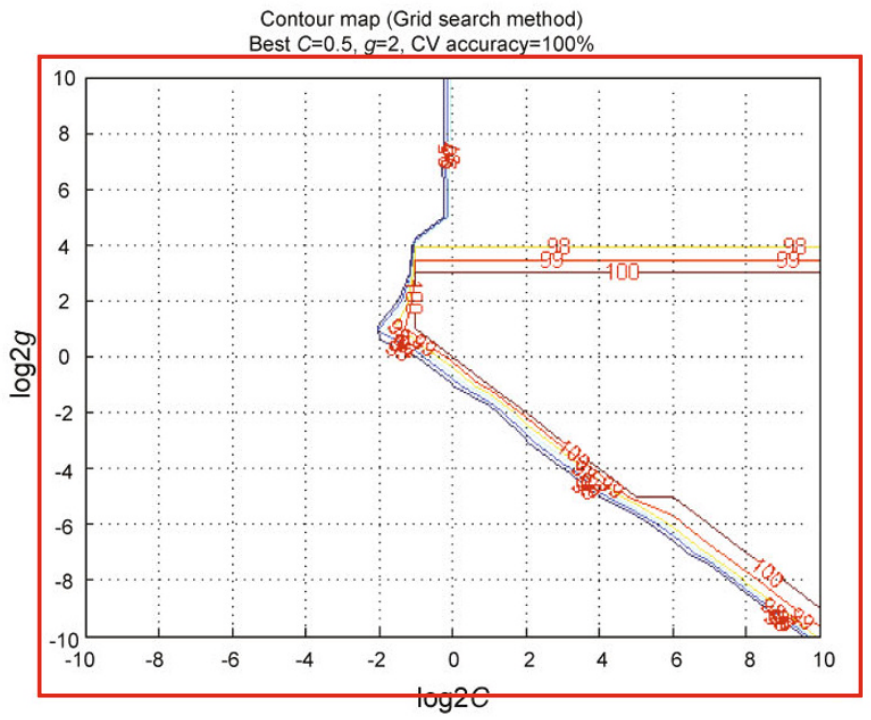

Fig. 7 Contour map of parameter results based on the CV (rough) model

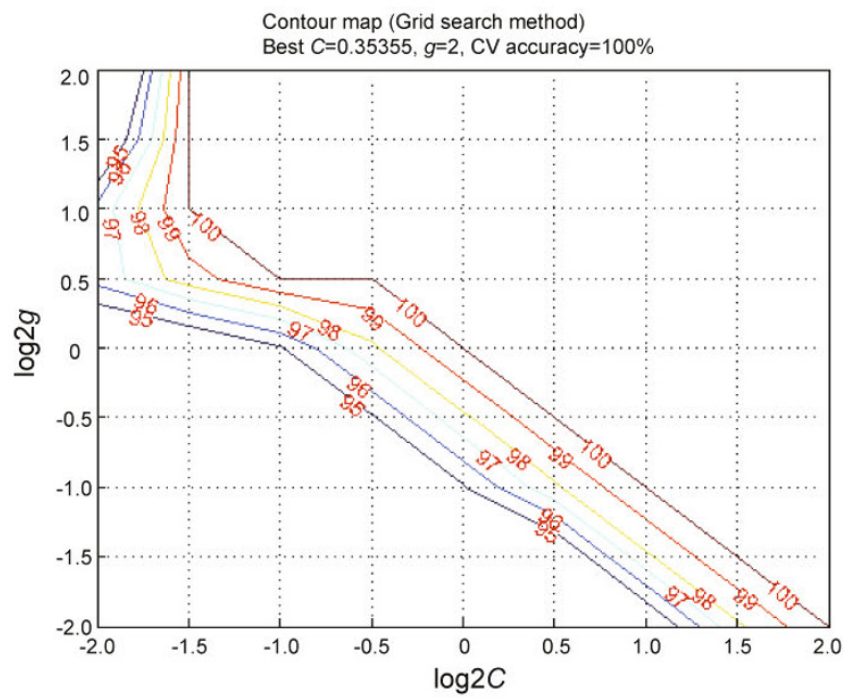

Fig. 8 Contour map of parameter results based on the CV (fine) model

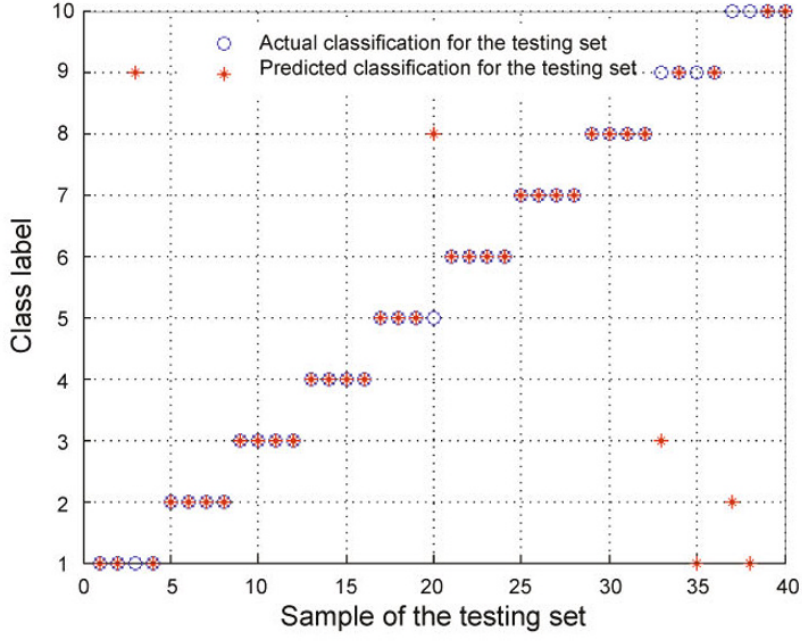

Fig. 9 Actual classification and predicted classification of testing samples with $C=0.35355, g=2$

maximum and minimum values of the penalty factor $C$ are $10^{4}$ and $10^{-1}$ respectively; the maximum and minimum values of the kernel function parameter $g$ are $10^{2}$ and $10^{-2}$ respectively; the classification accuracy rate of the testing set is chosen as the fitness function.

88 training samples and 40 testing samples are used as training samples and testing samples of the SVM.

Finally, the best parameters $C=15$ and $g=2.3$ are obtained after iteration.

The fitness function curve and the classification results of the training samples and testing samples are shown in Figs. $10-12$, respectively.

The following conclusions are drawn from simulation results: the parameters $C$ and $g$ selected by the PSO algorithm are used as the parameters of SVM and the classification accuracy rate of the training samples has always been kept at a high level, and more important is that the classification accuracy rate of the testing samples increases from $85 \%$ to $98 \%$. Thus, compared to the conventional CV method, using the PSO algorithm to select parameters not only

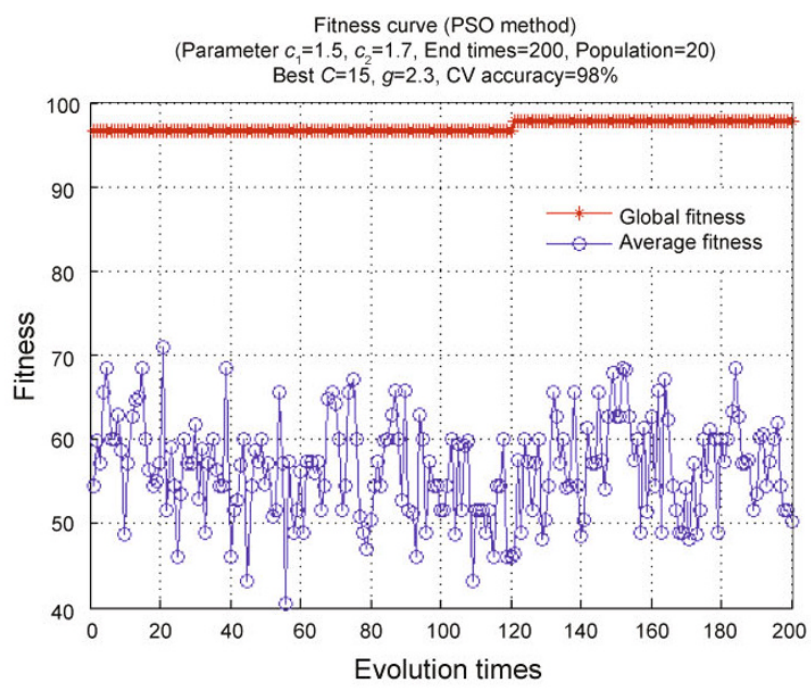

Fig.10 Fitness function 


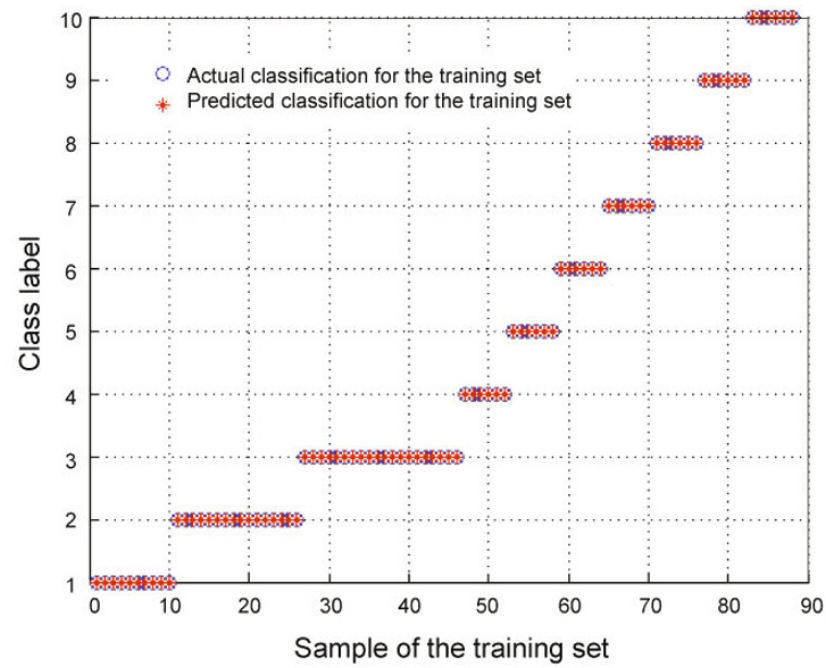

Fig. 11 Actual classification and predicted classification of training samples with $C=15, g=2.3$

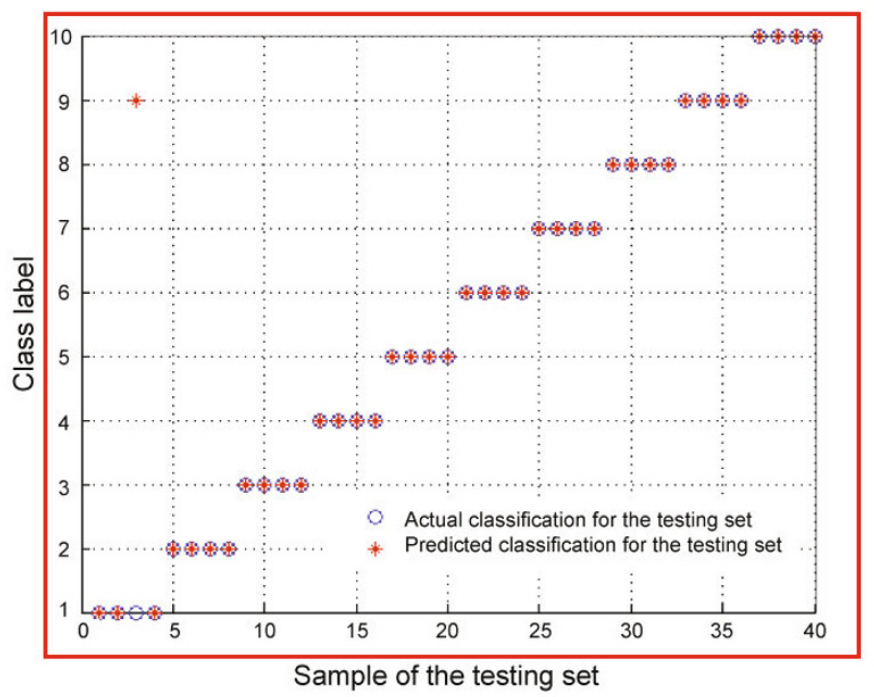

Fig. 12 Actual classification and predicted classification of testing samples with $C=15, g=2.3$

ensures the learning ability of the SVM but also increases the generalization ability of the SVM, thus improving the classification ability of the SVM on the whole.

\section{Conclusions}

This paper proposes a method based on the curve moment and PSO-SVM for fault diagnosis of sucker rod pumping units. The downhole dynamometer card is divided into four parts according to the working principle of the rod pumping unit. Curve moment parameters of each partition are extracted according to the curve moment theory, and 28 feature vectors with certain geometric significance can accurately describe different characteristics of the downhole dynamometer card in an oil rod pumping unit. The SVM method is used in this paper for pattern classification, which uses the curve moment parameters of different typical dynamometer cards as learning samples. The PSO algorithm is used to find the best parameters $C$ and $g$ in order to improve the classification efficiency of the SVM. The simulation results show that the curve moment and the PSO-SVM method proposed in this paper has better classification ability to diagnose working conditions of sucker rod pumping units based on the dynamometer card analysis.

\section{Acknowledgements}

Financial support from the Key Project of the National Natural Science Foundation of China (61034005) and Postgraduate Scientific Research and Innovation Projects of Basic Scientific Research Operating Expenses of Ministry of Education (N100604001) and Excellent Doctoral Dissertations Cultivation Project of Northeastern University is acknowledged. We also thank for the support from the Liaohe Oilfield of China, providing us research and experimental conditions.

\section{References}

Campbell W M, Campbell J P, Reynolds D A, et al. Support vector machines for speaker and language recognition. Computer Speech \& Language. 2006. 20(2): 210-229

Chapelle O, Vapnik V, Bousquet O, et al. Choosing multiple parameters for support vector machines. Machine Learning. 2002. 46(3): 131159

Chen C C. Improved moment invariants for shape discrimination. Pattern Recognition. 1993. 26(5): 683-686

Chen J L. A fast algorithm for down-hole dynagrams in sucker rod pumping wells. Acta Petrolei Sinica. 1988. 9(3): 105-113 (in Chinese)

Chowdhury S, Sing J K, Basu D K, et al. Face recognition by generalized two-dimensional FLD method and multi-class support vector machines. Applied Soft Computing. 2011. 11(7): 4282-4292

Derek H J, Jennings J W and Morgan S M. Sucker rod pumping unit diagnostics using an expert system. Paper SPE 17318 presented at Permian Basin Oil and Gas Recovery Conference, March 10-11, 1988, Midland, Texas

de Souza A, Bezerra M and Filho M, et al. Using artificial neural networks for pattern recognition of downhole dynamometer card in oil rod pump system. Proceedings of the 8th WSEAS International Conference on Artificial Intelligence, Knowledge Engineering and Data Bases, February, 2009, Cambridge, England, United Kingdom, Pages 230-235

Eberhart R C and Kennedy J. A new optimizer using particle swarm theory. Proceeding of the 6th International Symposium on Micro Machine and Human Science, October 4-6, 1995, Nagoya

Ganapathiraju A, Hamaker J E and Picone J. Applications of support vector machines to speech recognition. IEEE Transactions on Signal Processing. 2004. 52(8): 2348-2355

Gibbs S G and Neely A B. Computer diagnosis of down-hole conditions in sucker rod pumping wells. Journal of Petroleum Technology. 1966. 18(1): 91-98

He Y F, Wu X D, Han G Q, et al. Frequency spectrum analysis method for recognition of dynamometer card. Acta Petrolei Sinica. 2008. 29(4): 619-624 (in Chinese)

Kennedy J and Eberhart R C. Particle swarm optimization. IEEE International Conference on Neural Networks, November 27-December 1, 1995, Perth, Western Australia, Australia

Li J Y, Chen G C, Li Z F, et al. Application of filter technique to diagnosis of sucker-rod pumping system. Acta Petrolei Sinica. 2010. 31(1): 144-147 (in Chinese)

Li Y H, Xia J B, Zhang S L, et al. An efficient intrusion detection system based on support vector machines and gradually feature removal 
method. Expert Systems with Applications. 2012. 39(1): 424-430

Li Z, Liu B, Liu T N, et al. The research on fault diagnoses of oil pump based on support vector machine. Control \& Automation. 2006. 22(3): 1-3

Liang H and Li X M. Accurate extraction of valve opening and closing points based on the physical meaning of surface dynamometer card. Petroleum Exploration and Development. 2011. 38(1): 109-115 (in Chinese)

Martinez E R, Moreno W J, Castillo V J, et al. Rod pumping expert system. Paper SPE 26246 presented at SPE Petroleum Computer Conference, July 11-14, 1993, New Orleans, Louisiana, USA

Mountrakis G, Im J H and Ogole C. Support vector machines in remote sensing: A review. ISPRS Journal of Photogrammetry and Remote Sensing. 2011. 66(3): 247-259

Nath J S and Shevade S. An efficient clustering scheme using support vector methods. Pattern Recognition. 2006. 39(8): 1473-1480

Rogers J D, Guffey C G and Oldham W J B. Artificial neural networks for identification of beam pump dynamometer load cards. Paper SPE 20651 presented at Annual Technical Conference and Exhibition, September 23-26, 1990, New Orleans, Louisiana

Shi W G, Dai J J and Wang Y. FSVM based recognition approach for downhole pump dynamometer cards. Oil Field Equipment. 2004. 33(4): 46-48 (in Chinese)

Shi Y and Eberhart R C. A modified particle swarm optimizer. IEEE International Conference on Evolutionary Computation Proceedings, IEEE World Congress on Computational Intelligence, May 4-9, 1998, Anchorage, Alaska, USA

Tian J W, Gao M J, Li K, et al. Fault detection of oil pump based on classify support vector machine. 2007 IEEE International Conference on Control and Automation, May 30-June 1, 2007a, Guangzhou, China

Tian J W, Gao M J, Liu Y X, et al. The fault diagnosis system with selfrepair function for screw oil pump based on support vector machine. Proceedings of the 2007 IEEE International Conference on Robotics and Biomimetics, December 15-18, 2007b, Sanya, China

Wang J P and Bao Z F. Study of pump fault diagnosis based on rough sets theory. 2008 3rd International Conference on Innovative Computing Information and Control (ICICIC), June 18-20, 2008, Dalian, Liaoning, China

Wang M H, Zhang H B and Ding R S. Research of text categorization based on SVM. International Conference on Informatics, Cybernetics, and Computer Engineering, November 19-20, 2011, Melbourne, Australia

Wolfson H J. On curve matching. IEEE Transactions on Pattern Analysis and Machine Intelligence. 1995. 12(5): 483-489

Widodo A and Yang B S. Application of nonlinear feature extraction and support vector machines for fault diagnosis of induction motors. Expert Systems with Applications. 2007. 33(1): 241-250

Wu W, Sun W L and Wei H X. A fault diagnosis of sucker rod pumping system based on wavelet packet and RBF network. Advanced Materials Research. 2011. 189-193: 2665-2669

Xu P, Xu S J and Yin H W. Application of self-organizing competitive neural network in fault diagnosis of sucker rod pumping system. Journal of Petroleum Science and Engineering. 2007. 58(1-2): 43-48

(Edited by Sun Yanhua) 